\title{
Role of $n-6$ PUFA derived lipid mediators on epithelial barrier function in intestinal Caco-2 cell monolayers
}

\author{
M. J. Rodríguez-Lagunas, R. Martín-Venegas, J. J. Moreno and R. Ferrer \\ Departamento de Fisiologia, Facultat de Farmàcia, Universitat de Barcelona, Av. Joan XXIII s/n, Barcelona and Institut \\ dInvestigació en Nutrició i Seguretat Alimentaria
}

The intestinal epithelium forms a regulated and selectively permeable barrier that allows transcellular and paracellular passage of luminal nutrients, water and ions, but restricts the access of potentially harmful substances. Several intestinal diseases are associated with the disruption of epithelial barrier function, particularly inflammatory bowel disease (IBD) ${ }^{(1)}$. Although it is not clear whether intestinal barrier dysfunction is involved in the initiation or is the result of intestinal inflammation, it is likely that this dysfunction may perpetuate the stimulation of the mucosal immunity. In fact, epithelial cells are recognized to play a key role in the innate and adaptive immune responses.

Eicosanoids are lipid mediators synthesized from membrane arachidonic acid (n-6 PUFA) through three distinct pathways: cyclooxygenase (COX), lipooxygenase (LOX) and cytochrome $\mathrm{P} 450$ pathways. We recently demonstrated that the addition of prostaglandin $\mathrm{E}_{2}$ $\left(\mathrm{PGE}_{2}\right)$ to differentiated Caco-2 cells induces the disruption of epithelial barrier function ${ }^{(2)}$. Moreover, this eicosanoid is increased in the mucosa of IBD patients ${ }^{(3,4)}$. The objective of this study was to investigate in differentiated Caco- 2 cell monolayers, the potential of different eicosanoids to disrupt paracellular permeability (PP). For this purpose, the cells were grown on polycarbonate filters with a pore size of $0.4 \mu \mathrm{m}$ (Transwell ${ }^{\mathbb{R}}, 4 \times 10^{5}$ cells $/ \mathrm{cm}^{2}$ ) for 21 days. PP was assessed from transepithelial electrical resistance (TER) and apical to basolateral D-mannitol fluxes $\left(0.4 \mu \mathrm{Ci} / \mathrm{ml} \mathrm{D}-\left[2-{ }^{3} \mathrm{H}\right]\right.$ mannitol $)$ in cultures maintained for $3 \mathrm{~h}$ with different eicosanoids in the apical and basolateral compartments.

In contrast to $\mathrm{PGE}_{2}, \mathrm{PGD}_{2}$, another $\mathrm{COX}$ pathway derived product, did not modify PP. As for the LOX pathway, 5-, 12- and 15-hydroxyeicosatetraenoic acids (5-, 12(R)-, 12(S)- and 15-HETE) increased PP although 12(R)- and 12(S)-isomers did not change TER. Another LOX metabolite, leukotriene $\mathrm{B}_{4}\left(\mathrm{LTB}_{4}\right)$ did not exert any effect, whereas $\mathrm{LTD}_{4}$ increased D-mannitol fluxes without changes on TER. Finally, the cytochrome P450 products, 11,12- and 14,15- epoxyeicosatrienoic acids (11,12- and 14,15-EET) did not alter epithelial barrier function.

Table 1. Effect of eicosanoids on PP expressed as percentage of the treatment condition with respect to the control condition (means \pm SEM)

\begin{tabular}{lcr}
\hline Eicosanoids & D-mannitol fluxes & TER \\
\hline Control & $100 \pm 7.71$ & $100 \pm 1.62$ \\
PGE $_{2}(3 \mathrm{nM})$ & $152.83 \pm 8.49^{*}$ & $83.83 \pm 3.16^{*}$ \\
5 -HETE $(100 \mathrm{nM})$ & $340.90 \pm 59.32^{*}$ & $70.76 \pm 9.79^{*}$ \\
12 -(R)-HETE $(100 \mathrm{nM})$ & $275.29 \pm 28.27^{*}$ & $107.67 \pm 2.80$ \\
$12-(\mathrm{S})$ - HETE $(100 \mathrm{nM})$ & $170.50 \pm 31.00^{*}$ & $108.56 \pm 5.01$ \\
$15-\mathrm{HETE}(100 \mathrm{nM})$ & $312.09 \pm 84.51^{*}$ & $86.58 \pm 4.11^{*}$ \\
LTD $_{4}(50 \mathrm{nM})$ & $139.78 \pm 9.60^{*}$ & $93.83 \pm 6.87$ \\
\hline
\end{tabular}

$* P<0.05 v$. control $\left(11.67 \pm 0.79 \mathrm{fmol} / \mathrm{cm}^{2}, 200.39 \pm 2.63 \Omega . \mathrm{cm}^{2}\right)$.

In conclusion, we have demonstrated that different eicosanoids are able to disrupt epithelial barrier function leading to the diffusion of luminal solutes as well as pathogens and toxins through the intercellular space thus triggering mucosal immune responses.

This study was supported by grant BFU2007-61727/BFI (Ministerio de Ciencia y Tecnología) and 2005SGR0269 (Generalitat de Catalunya).

1. Turner JR (2006) Am J Pathol 169, 1901-1909.

2. Martin-Venegas R, Roig-Perez S, Ferrer R et al. (2006) J Lipid Res 47, 1416-1423.

3. Hommes DW, Meenan J, de Haas M et al. (1996) Gut 38, 564-567.

4. Berg DJ, Zhang J, Weinstock JV et al. (2002) Gastroenterology 123, 1527-1542. 